\title{
Patient-centered outcomes: a qualitative exploration of patient experience with electroencephalograms in the Emergency Department
}

\author{
Christine S. Davis, ${ }^{1}$ Sandra K. Beverly, ${ }^{2}$ Jackeline Hernandez-Nino, ${ }^{2}$ Andrew J. Wyman, ${ }^{2}$ Andrew W. Asimos ${ }^{2}$ \\ ${ }^{1}$ University of North Carolina at Charlotte, Department of Communications; ${ }^{2}$ Carolinas Healthcare System, Department of Emergency \\ Medicine, Charlotte NC, USA
}

\begin{abstract}
The primary objective of this qualitative project was to understand the experience of patients who had first-time seizures and who did, and did not, have electroencephalograms (EEGs) performed in the Emergency Department (ED) as part of their initial evaluation, so as to refine the diagnostic and therapeutic approach to these patients and transform the standard of care for first-time seizures by focusing on outcomes as defined by patient experiences and expectations. In this paper, we show that, regardless of the diagnostic and therapeutic approach patients are given in the ED, patients and caregivers trust that health care providers will perform the standard of care consistent with the current medical practice for first-time seizures. However, performing EEGs in the ED and initiating appropriate anticonvulsant therapy for those patients who are at high risk for future seizures addresses patient needs by offering patients a sense of security and control over their medical condition and expediting appropriate follow up care, as long as clearly stated written diagnostic, treatment, and referral instructions are provided upon discharge.
\end{abstract}

\section{Introduction}

Seizures are fear-inducing experiences for those having them and for their friends and family members. ${ }^{1-3}$ Seizures comprise an estimated $1 \%-2 \%$ of all Emergency

Correspondence: Christine S. Davis, University of North Carolina at Charlotte, Department of Communications, 9201 University City Blvd, Charlotte, NC 28223, USA.

E-mail: Csdavis2@uncc.edu

Key words: First-time seizure, healthcare, electroencephalograms.

Contributions: all authors contributed equally.

Conflict of interest: the authors declare no potential conflict of interest.

Funding: this work was supported by an Emergency Medicine Foundation 2014-2015 Resident Research Grant and a Carolinas Healthcare System Internal Grant.

Conference presentation: this study was presented at the ACEP annual meeting in October of 2015 in Boston, Massachusetts.

Received for publication: 4 August 2016.

Revision received: 10 January 2018.

Accepted for publication: 10 January 2018.

This work is licensed under a Creative Commons Attribution NonCommercial 4.0 License (CC BY-NC 4.0).

CCopyright C.S. Davis et al., 2017

Licensee PAGEPress, Italy

Qualitative Research in Medicine \& Healthcare 2017; 1:81-92

doi:10.4081/qrmh.2017.6219
Department (ED) visits per year. ${ }^{4}$ Despite that fact that patient-centered outcomes and shared decision-making models are widely promoted in healthcare today, no research has attempted to understand the ED experience from the point of view of first-time seizure patients and their families, including their experiences of the setting and pace of the typical components of a first-time seizure evaluation, diagnosis and treatment.

The current ED practice standard for uncomplicated first time generalized seizures in adults evaluates patients for toxic, metabolic, structural, cardiogenic and systemic causes. ${ }^{5}$ If no abnormalities are identified, patients are instructed to refrain from at-risk activity (e.g. driving, swimming) and told to arrange follow-up with a neurologist. Since the standard neurologic evaluation for first-time seizures includes performance of an electroencephalogram (EEG) as an outpatient, patients must undergo an EEG after ED discharge. ${ }^{5,6}$ Research has demonstrated the importance of performing early EEGs among first-time seizure patients for timely identification of epilepsy, as rapid diagnosis and immediate initiation of antiepileptic drug (AED) therapy after an epileptic seizure significantly reduces seizure risk in the short term by about 35\%. ${ }^{5,7}$ However, the barriers to appropriate outpatient follow-up and the important patient and family centered outcomes related to first time seizures in adults are poorly understood.

In this paper, we utilize the concept of patient-centered outcomes to consider the patients' and families' experiences when deriving treatment options for first-time seizure patients.

\section{EEGs in the Emergency Department}

There has been virtually no research on Emergency 
Department (ED) experiences or post-ED experiences among patients with seizures, and little research examining the effects of EEG performance in the ED after a firsttime seizure. EEGs are a valuable tool to evaluate the functional status of the brain. Researchers have determined that first time seizures are related to an epilepsy diagnosis in $15 \%-47 \%$ of cases based on EEG testing ${ }^{8,9}$ As a case-in-point, researchers conducting a recent study of ED EEG performance found an epilepsy rate of $21 \%$ in adult first-time seizure patients who were candidates for discharge from the ED. ${ }^{10}$ In this study, all of these patients were started on AED therapy immediately upon discharge and followed up by a neurologist in two weeks. Conversely, first time seizure patients who are discharged from the ED without undergoing an EEG (and who ultimately have undiagnosed epilepsy) are at risk for recurrent seizures. In addition, the resulting delay in following up with a neurologist and in beginning anticonvulsant therapy is also medically problematic. For some patients, an inability to follow-up with a specialist after being discharged from the ED results in substantially delaying or entirely neglecting appropriate follow-up care. ${ }^{10}$

EEG use in the ED is often limited by the availability of trained staff, time limitations, and financial constraints. Yet, physicians are better able to diagnose and treat patients when they have the EEG results in hand. For example, researchers examining the use of EEGs in the management of patients who present with altered mental status reported that an EEG helped to establish the diagnosis in $56 \%$ of the cases, changed diagnostic management in $49 \%$ of cases, and changed treatment plans in $42 \%$ of the cases. ${ }^{11}$ Similarly, Praline, Grujic, Corcia, and colleagues ${ }^{12}$ note that EEGs ordered on an emergent basis in the ED as well as in the hospital contributed to the diagnosis in $78 \%$ of cases examined. Sadleir and Ingrid ${ }^{13}$ report in a study performed on children presenting with first-time seizures that the rate of epileptiform discharge (uncontrolled electrical discharges in the brain associated with epilepsy, identified through EEGs) was $57 \%$ in patients on an EEG performed within 24 hours of the seizure, and another study of EEGs performed in adults and children within 24 hours of a firsttime seizure found a rate of epileptiform discharge of $47 \%{ }^{9}$ Importantly, this study demonstrates that early EEG performance had a higher rate of finding epilepsy (51\%) compared to $34 \%$ when EEGs are performed later. The use of EEGs in the ED for seizure patient management is a feasible option if utilized properly. ${ }^{14,15}$ EEGs administered in the $\mathrm{ED}$, and remotely interpreted by an on-call epileptologist, have been found to allow providers to initiate appropriate therapeutic treatment earlier as well as arrange for close outpatient follow up. ${ }^{10}$

\section{Patient-centered outcomes research}

In the patient-centered outcomes and shared decisionmaking model widely promoted in healthcare today and mandated by the 2010 Affordable Care Act, patients and families actively participate in their own treatment decision making and planning. Patient-centered care is a model of shared power between the patient and the medical provider ${ }^{16}$ defined as care that is respectful and responsive to individual patient preferences, needs, and values and customizes treatment recommendations on the basis of informed, shared decision-making [...] development of patient knowledge, enhancement of skills needed for self-management of illness, and preventive behaviors. ${ }^{16}$

Researchers studying patient-centered care have concluded that including patients and families as partners in the decision process enhances clinical outcomes and treatment compliance, leads to more cost-effective healthcare, and is a more ethical choice. ${ }^{17-21}$ However, balancing evidence-based medicine with patient choice is a challenge. ${ }^{17}$ Two keys to implementation of this model are effective patient-provider communication and information management. ${ }^{16,17,22}$ Essential to this model is the inclusion of patient and family experiences, needs, and perspectives, including sources of distress, social issues, and behavioral issues: ${ }^{16}$ with therapeutic information and knowledge, and by aligning medical decisions with health outcomes that are relevant to patients and families. ${ }^{18,20,22}$ The concept of patient-centered outcomes is one of shared voice-patients and families being heard, respected, understood, and considered by healthcare providers. ${ }^{18,20,22} \mathrm{~A}$ focus on patientcentered outcomes does not preclude clinical or therapeutic considerations, but this focus by definition also incorporates consideration of quality of life, access to care, and patient preferences. ${ }^{18,22,23}$ So important is this concept of patient-centered outcomes that the Patient-Centered Outcomes Research Institute (PCORI) - created in 2010 by the ACA - supports extensive research in this area. ${ }^{19,20}$

\section{Study objectives}

The primary objective of this qualitative project is to understand the experience of patients who had first-time seizures and who did, and did not, have EEGs performed in the ED as part of their initial evaluation, so that the approach to their care could be refined. This study is part of a larger project focusing on both quantitative and qualitative outcomes defined by patient experiences and expectations. In all of this related research, the overarching goal is to transform the standard of care for first-time seizure patients to improve the experience for both patients and providers.

In this portion of the research, we sought to specifically address the following Research Questions:

RQ1: What was the experience like (both in the ED and after) for patients and families following $a$ first-time seizure experience?

RQ2: What are the important patient outcomes following a first-time seizure experience?

RQ3: What are patients' and families' expected standards of care for treatment of first-time seizures in the ED? 


\section{RQ3: How might patients and families' experiences of first-time seizures be shaped by performance of EEGs in the ED?}

\section{Materials and Methods}

Focus groups are a qualitative research tool that capitalizes on group processes and interpersonal interactions to gain rich experiential data about a topic of interest. ${ }^{24-27}$ Focus groups bridge the emic-naturalistic data and the etic-researcher imposed data, and are especially useful for understanding the patient experience and determining patient centered outcomes as they are discussed in a group setting. ${ }^{27}$ We conducted four homogeneous focus groups among i) patients with seizures who had an EEG performed in the ED; ii) patients with seizures who did not have an EEG performed in the ED; iii) family members of adult patients recently diagnosed with seizures; iv) seizure patient advocates. The focus groups each had between three and nine participants, for a total of 22 focus group participants. We attempted to hold one additional group with patients with seizures without health insurance coverage but only one participant attended that session, so we conducted a personal in-depth interview with her instead.

We did have a significant no show rate for some of the focus groups, so, following the focus groups, we conducted seven telephone interviews to increase diversity in the sample and to further inform study findings about experiences of patients with no health insurance and nonEnglish speaking Hispanic first-time seizure patients.

In all the focus groups and interviews, we gathered information about: i) perceptions of the ED experience; ii) perceptions of the experience obtaining, or not obtaining, an EEG in the ED; iii) perceptions of treatment during and after the ED; iv) their experience of the follow-up care; v) perceptions of the experiences after treatment; vi) desired outcomes for treatment (during and after); vii) perceptions of factors that facilitated or hindered follow-up care; and viii) perceptions about the care in general.

The groups and interviews were facilitated by the first author who is a university researcher, the second author who was an emergency medicine resident, and the third author who is an Emergency Medicine Researcher and is proficient in Spanish. We audio and video recorded the focus group sessions and audio recorded the interviews. Each session lasted approximately 90 minutes.

\section{Recruiting and sampling}

Using purposive and convenience sampling, we recruited participants from the medical center's ED and local neurology and epilepsy clinics. Participants were current or former patients of referring physicians, were family members of current or former patients, were participants in a prior first-time seizure research project who indicated agreement to participate in future research, or were seizure patient advocates recruited from a local epilepsy center.

Specifically, the focus group and interview participants (and their family members) who had an EEG performed in the ED were recruited from an ongoing quantitative research project studying EEG performance in first-time seizure patients. ${ }^{10}$ The focus group and interview participants (and their family members) who did not have ED EEGs performed were recruited from a regional healthcare system's neurology and epilepsy clinics. We recruited seizure patient advocates from local and regional epilepsy support groups.

The focus group and interview participants who had an EEG performed in the ED and who were recruited from the ongoing quantitative research project studying EEG performance in first-time seizure patients had a follow up appointment made for them before they were discharged from the emergency department and - as a result of being enrolled in the ongoing quantitative study - were guaranteed the opportunity to follow up with a neurologist within two weeks of their ED visit irrespective of insurance status. We do note that this may have influenced their perceptions about the impact of an ED EEG in facilitating follow-up.

There were five individuals who participated in the focus group for patients had who had an EEG in the ED. All five of these individuals were insured. Four of these individuals were diagnosed with epilepsy based upon the EEG that they received in the ED. All of these individuals were started an antiepileptic medication. The remaining individual had an abnormal EEG but was not diagnosed with epilepsy and this person was not started on medication. The five interview participants who had an EEG in the ED were not insured, as we purposely sought out noninsured patients for these interviews to gain an additional perspective we felt was missing from the focus groups alone. One of those five was a non-English speaking woman of Hispanic ethnicity who was purposively included for her ethnic perspective. All of these participants had normal EEGs and were not started on antiepileptic medications.

There were three participants in the focus group for those patients who did not have an EEG in the ED. In addition, one of the telephone interviews was with a patient who did not have an EEG in the ED.

Three family members and two patients participated in the focus group for family members. Family members included a father, a sister and a significant other. One of the two patients in this group did not have an EEG in the ED and the other did have an EEG in the ED which was read as epileptic, but the patient was not started on an antiepileptic medication. In addition, the phone interviews included one family member of a person who did not have an EEG in the ED and did not have health insurance.

There were a total of nine participants in the focus 
group for advocacy members, which included the director of the local epilepsy foundation, family members of patients with epilepsy, and patients who were diagnosed with epilepsy. As we stated, we conducted an interview with the one patient who attended the focus group for the patients who did not have insurance.

All study participants were age 18 and older and were able to consent. We gave participants 15 US dollars (\$) gift cards for their participation and provided them with a meal during the focus group sessions. Our study was approved by the Institutional Research Boards of Carolinas Healthcare System and the University of North Carolina at Charlotte, and we reviewed informed consent with participants when they were invited, individually when they arrived for the focus group session, and again as part of the introductory comments in the session.

\section{Coding and analysis}

Utilizing Charmaz' ${ }^{28}$ emergent approach to research, analysis and questioning began early in the process and continued throughout all stages of research. We debriefed possible findings after each focus group session and revised the facilitation guide as the group sessions progressed. We added the seven telephone interviews to address questions and gaps that emerged as we moved through the data collection and analysis stages. Of course, focus groups themselves are a non-hierarchical method in which participant voice is more equalized than in positivist or social science based methods, and in which participant construction of meaning is key, ${ }^{26}$ so the groups themselves represented a partnership between study participants and researchers to make meaning of the experience. We transcribed all audio and videotapes word-for-word indicating conversational turns and speaker changes. We coded and analyzed data by means of Charmaz' ${ }^{28}$ constructivist approach to grounded theory coding, utilizing a systematic, multi-step coding process starting with line-by-line coding and employing the constant comparison method to derive categories and themes. This is an inductive approach to data analysis, which starts with a broad research question and narrows down to understanding a phenomenon based on the data itself.

We established a qualitative approach to validity and reliability with the use of multiple coders and a systematic process in which all five members of the research team each coded the transcripts individually, first making multiple passes through the transcripts to immerse ourselves in the data, jotting down analytical notations, including initial thoughts and ideas. We then each individually moved from this stage to development of a list of potential themes and ideas. The research team then met and compared and debriefed their initial analytical ideas. As a group, we created an initial list of metacodes and thematic categories which represented the focus group and interview findings. The first two authors then made additional multiple passes through the data to further refine the cat- egories and connect the transcript text to each code through the constant comparison process by looking for similarities and differences in the data and the emerging categories. We compared each emerging category with each other category and combined, divided, and sorted the data into final themes until all of our data fit into categories. ${ }^{29}$ The results reported here represent the final categories and are illustrated with representative quotes from the transcripts.

\section{Results}

Seizures are experienced as traumatic emergencies and, coupled with the memory loss and confusion firsttime seizure patients experience surrounding the ED visit due to their medical state at that time, for the majority of patients, this experience is fear inducing, unknown, unexpected, and stigmatizing. In addition, for some patients, the first-time seizure experience has negative financial ramifications, adding to the trauma of the experience. The medical testing, treatment, and discharge experience in the ED is confusing to patients. They don't understand or clearly remember what they are told in the ED. In addition, referral information is inadequately provided and too long a wait for follow-up care, resulting in an anxiety-provoking experience. Regardless of the diagnostic measures offered to patients in the ED, for the most part, patients trust medical professionals to act in their best interest during this time; however, patient experiences suggest that earlier diagnostic testing and provision of medication and specialist referrals would ease patient distress, as long as clearly stated written diagnostic, treatment, and referral instructions are provided upon discharge. Detailed discussion of the categorical findings follows.

\section{Seizures are experienced as traumatic emergencies}

\section{Experience is traumatic, fear inducing and stigmatizing}

The seizure experience is completely unexpected and sudden, occurring sometimes at night, sometimes in public or when around others, often in dangerous, terrifying, embarrassing, or stigmatizing situations. The seizure itself does not last long, although the overall experience might feel interminable. Participants describe a chaotic, out of control experience, compounded by physical incidents such as a bit tongue, loss of bladder control, agitation, dizziness, confusion, loss of consciousness, memory loss, vision loss, shaking, and incoherence. Participants emphasize undergoing emotions surrounding the seizure experience of feeling different, uneasy, and out of control. Memory loss coupled with mental confusion and lack of comprehension makes the experience all the more terrifying. Additionally, the seizure experience interrupts one's daily routine in many ways, both during and after the experience itself, further adding to the feelings of being out of control. For most first-time seizure patients, EMTs are the first line of medical 
care. EMTs transport patients to the ED, administer oxygen, check vital signs, and check for injuries, so the EMT response adds to the initial experience.

I had come in with my friend that I was at her house, and we were getting ready to go to bed. It was around 11 o'clock and she said she turned around to turn off the lights, turned back around and I was in a seizure.

Mine happened as I was driving but luckily I sensed that I was not feeling right, so I got to the CVS pharmacy to get something to eat and some water and walked in there, walked back to the cooler got a water, walked up to the counter and I was feeling light headed. My vision was really kind of blurry, and I could not focus and see. I remember it took me a minute to focus. I tried to find a candy bar, because I do have low blood sugar, so I thought that might be the reason, what the light headedness was. I could not focus, and the last thing I remember is punching my PIN code and that was it. Then I woke up in the emergency room.

I work uptown and at 5 o'clock I was coming home on Stonewall St. right up the hill and I was talking to my daughter and she said, 'Mom, something is wrong with you', and I was aware that I could not find the words to express, but I was kind of making a joke out of it, like 'What are you talking about, don't make fun of me', but I knew honestly something was really wrong and that is the last thing I remember. I totaled my car. I hit a couple of light posts and another car.

I was at the mall and I had my two-year-old with me and we were at Gymboree and I just remember this overwhelming sense that I am not okay, I need to tell somebody, and I could not speak, and then I woke up and I was on the floor, and hit my head and my shoulder was hurting. This sweetest teenage manager in that store was just looking at me and saying, I just remember looking up and she just said, 'It's okay, you had a seizure, help is on the way, 'and I remember being concerned about my son and there were all these moms playing with him and he was fine. I had programmed ICE into my phone, and so they had already called my husband and he was on his way, so they said, 'Your husband is on his way, the ambulance it's on the way, 'and then I don't remember a couple of hours.

I just remember looking across the store and then I remember being at the front of the store...I wanted to know what happened...I wanted to know who called you? How did I...how did I end up here?

I guess I took down some displays. And I was kind of fighting back. They put an IV in me and I knocked it out. I was just kind of going nuts. From talking to the EMS or the fire fighters they said that they really had to pump a lot of anti-seizure stuff to get me to calm down because I was just kind of going nuts. My face was kind of bruised up and I had a black eye for a couple days, looked like I was in a fight.

\section{Experience is unknown and uncertain}

While some participants have a family history of seizures and thus have some idea - at least after the fact - of what is happening, others have no prior knowledge of seizures. Some family members recognize the experience as seizures at the time of onset, but some do not know what is going on at the time of seizure. Regardless of prior knowledge, participants are quite unprepared for the experience.

My child had petit mal seizures so I knew grand mal could come, [but] I was not prepared for it when it happened.

I did not feel anything. I never felt anything when I had the seizure, but I have seen other people having a seizure and everything they went through. When I had the seizure, my husband was desperate.

As previously stated, the experience is extremely frightening. A large part of the fear is based on not knowing what is happening. Parents of children with seizures report being fearful that their child is dying, and the loss of control of oneself or one's child is especially terrifying.

When we [went into] into the emergency room it's scary, [my son is] two years old, you don't know what's going on, that was very scary... and at that point he came out of it...it's very traumatic.

That is trauma ... you think they died.

You hear people talk about this, it is one of the most frightening moments of their life.

My nine month old, I took her out of the car and her eyes rolled back...I went inside; I am freaking out, what do you think is wrong with her that her head started shaking, I started screaming, someone called 911, and they came.

\section{Experience results in confusion and memory loss}

Even more unsettling to the participants than not being able to remember what happened during the seizure itself is not being able to recall the information that is given during the ED stay. During the ED visit, participants undergo several tests in an attempt to decipher why they had the seizure. Prior to discharge, the patients are informed of their results, with or without someone else present. $\mathrm{Pa}$ tients are in no state of mind to understand this information at that time. For some, it is because of the overwhelming fear that they experience; for others, they are not able to understand simply because of the confused state of mind that lasts for hours to days after the seizure. Often, participants are not able to recall information about how they arrived at the ED, what happened in the ED, or even what the results of the ED tests showed. Further, patients are also unable to remember crucial details about follow up appointments or directions for prescription medications.

After I got back to work, I forgot my results, so I had to see the doctor...I made an appointment. He 
made me come and see him like in 10 days afterwards, and then I thought that it was the first I was meeting him. He said, 'No, because we talked in the emergency room.' I said, 'I don't remember', because I was taking my pills wrong. I was taking them backwards and he said, 'I told you this in the emergency room' and I said, 'Sorry'.

\section{Patients need reassurance in the ED}

Once in the ED, patients undergo numerous diagnostic tests in an attempt to decipher why they experienced a seizure, including CT-scans or brain scans, EEGs, MRIs, blood tests, and measurement of vital signs including blood pressure and temperature. Most participants have vague memories of medical testing, but many cannot recite which tests were completed.

Well I was certain there was [testing in the ED]; when I had the MRI I thought there was a brain tumor that is why, because they were all kinds of things going on, but it wasn't. I did all the follow up stuff, a whole battery of cardiac stuff just to rule that out, so I got every test done known.

They checked my blood...they were asking questions trying to induce, the strobe light thing you have to try to see if they could make one happen, and they are measuring your blood pressure and checking your pulse, you know the normal doctor stuff.

Most participants know that some testing was done in the ED but are not sure of what specific tests were completed.

They just did a, I guess it was an MRI, I don't know if they do an MRI or CT-Scan - what's the difference - just...to make sure no tumors were there.

The time course of the emergency department visit typically lasts several hours. The longer length of stay is commonly a result of physicians allowing the patients to awaken from their postictal state (the period of time after a seizure in which the patient is in an altered state of consciousness and confusion) following the seizure, as well as for allotting time to complete the required testing in the ED. Whether or not they have an EEG in the ED, participants are typically discharged from the ED that same day. This is the current standard disposition for an uncomplicated first time generalized seizure patient. Patients who have abnormalities identified on imaging or laboratory studies while in the ED sometimes require an overnight admission for further evaluation and management of the underlying issue. This includes additional laboratory testing or, for some patients, further brain imaging. Occasionally, a patient requires a second EEG to further elucidate what is happening with him/her. If admitted, the patient is typically seen in consultation by a neurologist.

Because seizures are not an infrequent experience to ED staff, and are not life-threatening conditions from a medical standpoint; but are terrifying and confusing to seizure patients and their families, there is a disconnect between the level of care and concern patients and family members need from the ED and the level of concern provided. Given that the patients are in the ED for many hours, there is a need for increased reassurance and information management.

I think I felt like there were lots of people in there. I never felt ignored, I never felt that people forgot about us, but I needed someone to say, I felt like [they acted as if] it was an everyday occurrence, and this was not an everyday occurrence in my life, and so I needed someone who would come in and say, we understand this is not an everyday occurrence so here is what we are going to do, you are going to follow up with your neurologist and your neurologist is going to give you, they are going to work with you on what other tests you need, and what medication you are going to need, and these are the steps, instead of just saying follow with your neurologist, just pitch it back in somebody else's hands.

Some patients report being placed in a hallway bed for several hours without anyone offering to help them change out of their soiled clothing. Some have traumatic injuries from the seizure and remember that no one helped to clean them up for a very long period of time. For some, the experience is so anxiety provoking, they require medication to calm down. In addition, some describe their experience as being treated nonchalantly by ED staff, in contrast to the perception of it being an extremely traumatic event for the seizure victims and their family members.

One of the things that I've heard from lots and lots of people over the years...it is one of the most frightening moments of their life, yet the emergency room staff see one of these every hour.

I went and had all the...they rush you down for the CT scan. You wake up and you have these little things on you [researcher note: electrodes]. You don't know why you have these things on you, who is doing what, but it is like, what do I do with this.

I am all bloody. Can somebody clean me up?

\section{Experience is overwhelming and anxiety-producing well after the seizure is over}

Participants experience a wide array of emotions after their discharge from the ED. Many recall being overwhelmed for several days after their first-time seizure and ED visit. After their seizure experience, patients take several days off of work and sleep for several hours a day to recover. The commonly reported emotion after the seizure experience is fear. Regardless of what happens in the ED, participants report lingering fear due to not fully understanding what having a seizure disorder means. Participants are afraid of the unknown and worried that they will have another seizure. Some participants are afraid to fall asleep afterwards because they are afraid that they will not wake up.

This was new for me. I never expected this of me and I was very depressed for a long time, very long 
time. I was crying all the time. I spent my time thinking and crying and wondering what was going to become of me. Why do I have to be medicated? But, thanks to a lot of people that I live with and especially my husband, his support and my friends'support, I started to understand the situation. I am not the only one that is going through this and there are worse things that people have to go through, worse than mine. And, little by little, I started understanding that my illness is not that bad. That, if I take care of myself, I am going to be okay.

Patients' baseline anxiety exists for a long time after the experience. Patients are fearful of experiencing more seizure activity and have thoughts about when and where another episode could occur. Some suffer from anxiety about bad outcomes if another seizure occurs. For many, a major restructuring of their confidence, self-esteem, and independence results from the seizure experience.

And you never know when it's going to happen again, and she is supposed to go on the eighth-grade swimming trip and I am saying, 'No you can't get in the ocean'. There is no way you know, and you do all this controlling stuff and you try to keep them from doing things and they wind up doing it anyway.

Before I felt confident, strong. Now I depend on the medicine to be well. If I do not take the medicine, I will have seizures again. And I am scared. That scares me because I have always been a very independent person all my life since I was very little, and I have to depend on people. I cannot be like that. That has also affected my personality because before I was always looking ahead. Now I just stay there, I don't do what I used to.

I was saying to that doctor, something is wrong with me. My husband was saying something was wrong with me. Everyone who works with me says, she just has this blank stare all the time, so I was relieved by knowing [that it was seizures], but it was like, what's going to happen next and how long could I go without.

To add to the levels of anxiety, their fears are not entirely unwarranted. Some patients do have recurring seizures.

She did have another seizure. They have said 50\% chance she would have another one and she had one a week later in the shower.

Patients want their lives to go back to normal as soon as possible after the seizure, and in an effort to reach normalcy, some try to stop thinking about the unknown and make efforts to manage the anxiety surrounding their diagnosis. Yet, most patients have to make significant changes to adapt to their seizure disorder, and participants note there are some unavoidable changes. All patients face undesirable lifestyle changes. For instance, participants are required to stop driving for at least six months following their seizure.

I lost my license promptly so that's that, but I said to my doctor, I cannot even think about driving again.
I mean it really scares me a lot, but I am almost on my six-month anniversary and I will be clear to go.

24 weeks counting until I can drive again.

I could not drive for 6 months so that really frustrated me practically because I wanted to drive when I wanted, where I wanted, and I became dependent on my husband practically all the time and I occupied his time that he needs for himself.

In addition to curtailing driving, patients also have to stop drinking alcohol post-seizure and some have to stop working. Some are instructed to avoid triggers such as caffeine and strenuous activities and exercising. Some patients curtail activities such as long car trips and vacations, and some refuse to go out alone. Participants report being treated differently by their family and friends following their seizure. In particular, family and friends are fearful of the seizures recurring. Some patients feel embarrassed and stigmatized by others. Understandably, participants report feelings of dependence on others and some patients feel so stigmatized they are reluctant to be around other people.

\section{Experience has negative financial ramifications for some patients}

Study participants included both insured and uninsured patients. For participants with health insurance, the majority of follow up and cost of medication is covered. For patients enrolled in the research trial portion of the related project, follow up care and medication cost were covered on a sliding scale basis. For participants who are not enrolled in the trial and who do not have health insurance, cost of follow up and medication is paid out-of-pocket. For patients not on health insurance, sometimes EMTs are the only line of emergency medical care; some uninsured participants report having declined ambulance transportation to the hospital because of the cost. In addition, some participants, particularly those without insurance, are unable to fill the prescriptions due to cost.

\section{Medical testing, treatment, and discharge is confusing and uninformative}

Some participants have EEGs conducted in the ED, and some do not. Regardless of what tests are conducted, much of the testing conducted in the ED does not indicate what is wrong with the patients, or comes back normal. This is not always the reassurance one might suppose it would be, because the patients are desperate for answers as to what has happened to them, and want indications that the ED staff takes their traumatic experience as seriously as they do. While sometimes the test results indicate abnormal brain activity or a possible diagnosis, and other results rule out other medical conditions such as a brain tumor, some test results from the ED are inconclusive. Participants describe being nervous leaving the ED without firm knowledge of what is wrong and what can be done. 


\section{Most patients are not discharged with medication/prescriptions}

Initiation of antiepileptic medication by an ED physician is not currently standard of care for the majority of first-time seizure patients, and patients are not typically started on medication prior to departure from the ED. If a patient has epilepsy, the longer time to initiation of antiepileptic medication can be dangerous and the patient is likely to experience a second seizure prior to outpatient follow up. As expected, this recurrence is frightening for patients and families and leads to a high degree of uncertainty.

You kind of go home with no medication from the ER...I am thinking, I am a mom, I am a working mom... What's next?

\section{Medications prescribed in the $E D$}

For patients who are prescribed medication in the ED, many take it as prescribed until the arranged follow up with a neurologist. However, other patients refuse the medication even though the epileptologist recommends it because of worry about potential side effects, fear that the $\mathrm{ED}$ is not the appropriate setting to start a new long-term medication, a lack of one-on-one interaction with a neurologist in the ED, and a disbelief that the medication will fix the underlying issue.

\section{Discharge from ED to home}

As we have stated, the majority of patients, whether or not they have an EEG, are discharged from the ED if the work-up is unremarkable. As part of the discharge process, patients are sent home with basic instructions for follow-up care and instructions on the conditions under which they should return emergently to the ED. Patient experience varies with regards to the discharge process. Some ED staff makes a clear effort to elucidate the discharge plan and provide instructions that patients perceive as clear and informative. Sometimes instructions are given to the patient him/herself, other times to a family member because of the patient confusion. Both patients and families can leave the ED with a clear explanation and clear course of action for discharge. However, for some patients and/or families, the discharge plan is confusing and perceived to be uninformative. Some participants leave the ED without an understandable explanation of what happened to them both during the seizure itself, and with regards to the ED stay.

There is not even a discharge paper that says seizure. What does that mean? You know, what is a seizure? You know, just what does this mean? Because I did not know, you know? You hear the word seizure. You hear the word epilepsy, but nobody knows what that is.

Everyone in the emergency room was very nice but I also remember feeling like, they are monitoring her to see to make she is okay, but then I leave not neces- sarily feeling any better because I do not have answers. So then it was like, I guess I won't go to the emergency room anymore.

Because you go home with lots of questions and then Lord have mercy you start googling and then it is all over.

\section{Referrals from ED are standard of care but anxiety inducing}

\section{Follow up appointments are a long wait}

When EEGs and other tests are conducted in the ED, participants are aware that the results are read by a doctor generally, or a neurologist or specialist specifically. For all participants who have an EEG in the ED, the test results lead to a follow-up appointment with a neurologist.

Moderator: Did you have an EEG while you were here?

Participant: Yes, I did, and I guess they noticed something little, in the left side of my brain, but I have not had the MRI yet to figure out if they can find the cause.

I had every test in the world done, and they all said I was fine, I knew that I was not okay.

Even for those with a relatively short period of time before their follow-up appointments, the idea of having to wait any period of time for more information evokes similar feelings of uncertainty and fear.

It is a long wait. When you can't get in somewhere right when you are waiting.

Participants understand that the purpose of a followup appointment is to further test for the cause of their seizure, and a follow-up with a neurologist seems like the natural next step for their condition after leaving the emergency department. Continued long-term follow up care after a first-time seizure does make participants feel comforted and reassured by having a physician tracking their medical issues on a regular basis. For those who are on medications, these treatments make their illnesses more manageable and are reassuring to patients.

[The treatments] offer me peace of mind because

I am not going through the scare that I will have another one.

Patients who had an EEG performed in the ED as part of the ongoing research project mentioned earlier includes adults who presented after an uncomplicated first time generalized seizure. Enrolled patients undergo a CT scan and a basic laboratory work-up in the ED. If this work-up is unremarkable, patients then have an EEG performed in the ED. The EEG is then immediately read by the on-call epileptologist (neurologist specializing in the treatment of epilepsy). Based upon the EEG findings, the epileptologist determines whether or not the patient has epilepsy warranting immediate initiation of antiepileptic drug therapy. If so, the patient is started on medication prior to discharge from the ED. All participants are discharged from the ED after the first-time seizure. These individuals also 
have a follow up appointment made for them before they are discharged from the emergency department and -as a result of being enrolled in that study - are guaranteed follow up in at least 2-3 weeks whether or not they have insurance. This follow up makes patients feel reassured and cared for. They know that although they had been through a traumatic experience, they are going to be followed upon on an outpatient basis.

The main discharge care was following up with the appointment with the neurologist.

The thing I would I remember is that he made a really strong attempt to get me in to see a neurologist quick. He could've just said, 'Oh, go home and rest, you did not have enough sleep, 'but that really was the most important thing, he did not try to diagnose as much as he said, 'Okay, I understand this is a neurological issue you need to get to see a neurologist.'

So I saw him 2 days later and I don't remember if they read the EEG that night and said any kind of on it anyway but I knew I would be right in to see him.

I think it was a month after I had the seizure. They ordered the exam and it was between two and three weeks. They found out that I have something wrong with the left side of my brain and that if it was going to get worse, something like that, and if the seizure were going to present more often then they would have to do surgery, but thank God for now I continue with the medicine. After that seizure I had in July, I had another one in December 2013, at that time I did not go to the emergency room, I went to the doctor.

In comparison, the majority of patients do not have an EEG in the ED and have to arrange outpatient follow-up on their own. This uncertainty makes those patients feel uneasy and anxious. To most, the main concern is the time between being discharged from the emergency department and the time to follow up with a neurologist. Often, this is weeks to months without an explanation for their ED presentation and without antiepileptic medications if the patient needs them.

Horrible. We had to wait for weeks as well. Actually, I don't think we were referred from the hospital. I remember going to my pediatrician and having a discussion of who I wanted, because I work with special needs kids and I knew neurologists. I was like, I want her and he was for it. I think that is where our referral came from, but also that in the interim time is when he was put on Depakote over the phone from the neurologist on call.

Some participants are also still struggling with medications months after the seizure experience. Some have issues with medication side effects requiring multiple medication changes while some are frustrated with the uncertainty of how long they will remain on medication, or if they will ever be able to be taken off.

The levetiracetam is what they gave me in emergency room and the doctor changed the medicine to another that did not depress me. This was the reaction from my body to the medicine.

They gave me something I don't remember what it was, I know the first drug I was prescribed by Dr. was Dilantin, and that made my gums swell very much. So then they stopped Dilantin and they went to one other one. I can't remember the name of that one. Now I am on carbamazepine.

\section{Patients expect standard of care to be performed in ED}

At the end of the focus groups, participants were asked a series of hypothetical questions in an attempt to better understand their reactions to potential diagnosis and treatment options in the ED. Participants who received an EEG in the ED were asked how they would feel if no testing was done in the $\mathrm{ED}$, and these participants believe that testing, including vital signs, blood work, head imaging, and EEGs, must be part of the standard of care for firsttime seizure patients. A lack of testing would have been unsettling and anxiety provoking and would have resulted in a lack of trust in the ED had no testing been completed following a first-time seizure.

Participants who were not prescribed medication in the ED were asked what they would have thought of receiving a prescription for antiepileptic mediation in the ED prior to discharge. These participants responded that because of the trauma of the seizure itself and the overwhelming confusion that existed in the ED, they would have been afraid and would have been unable to understand the medication instructions. These patients believe that the ED visit does not offer ample time for discussion of the medication and this would be more suited for an outpatient visit with a specialist who has the time to get a detailed history and who has the ability to adequately describe the medications to the patients. In addition, these participants would have wanted time to process what had happened to them before starting a medication. This response differs from that of participants who did receive medication prior to ED discharge who were reassured by being started on the medication in the ED. Interestingly, regardless of the testing performed or medication proscribed in the ED, all participants think their course of diagnostic testing and treatment was the most appropriate course of action.

In addition, all participants - regardless of their testing or medication status - strongly feel that, post-ED visit, rapid follow up with a specialist within two weeks is essential. If the wait for a specialist will be longer than that (and it was a months-long wait for some participants), even those participants who were reluctant to begin medication from an ED visit alone, prefer that to being sent home with a long wait for diagnosis and treatment. Even hesitant participants are more open to diagnosis and treatment in the ED if they are assured that it would be done in consultation with a specialist. 
In addition, the expectation for treatment in the ED is that that patients would receive rapid testing in the ED, including blood work, CT scan or MRI and an EEG. Some participants think that a neurologist should see a first-time seizure patient prior to discharge from the ED. Medication should be initiated promptly where it is indicated.

Well I think they got her in right away. What I remember, someone was talking to us all the time. I think is really important that people are seen quickly.

Anyone having seizures needs to be treated soon, they not need wait around for weeks or a year having seizures.

At first it's more important to get diagnosed properly, then to get on medications.

Regardless of their experience in the ED, most patients perceive the ED physicians, nurses, and staff to be caring, compassionate and gentle and perceive that a genuine effort was put forth to help the patient feel reassured and taken care of. The patients who do not speak English recall a clear effort to obtain an interpreter so that the patient and the family understood what was happening.

At the end of the focus groups and phone interviews, participants were asked their perceptions of standard of care for first-time seizure patients. The majority of participants were unsure of how to answer. Most patients trust that medical professionals will act in their best interest and give them the standard of care, whatever that is.

I trust in them. I trust in the doctor. I only listened to what they said that happened to me and I followed the recommendation of the specialist.

\section{Outpatient follow-up}

After ED discharge, first-time seizure patients typically require follow up with a neurologist. The outpatient follow-up allows for continued management of the patient's medications as well as a venue for arranging further testing if needed. For participants, neurology follow-up was typically very detailed and covered a much more detailed medical history than in the ED. This appointment was where the neurologist reviewed the work-up that had already been completed in the ED.

When I got the EEG in the ER, they had a group of nurses and I think a couple of doctors, going ahead and looking at what was done. They said that it looked normal to them. Afterwards we went to Dr. [epileptologist] and that's when he told us that there was [an abnormality], that if you weren't looking for it, you would not see it, but he saw it. It was one of the top lines. I don't remember which part of the brain that is and then he said it was on the left.

In addition, during follow up, additional CT scans, MRIs, EEGs, blood work, and other testing were completed. Similar to the ED visit, participants brought family and/or friends to the outpatient visit. The general theme is that the outpatient neurology visit was where participants felt most understood and reassured, and where they were better able to listen to and understand their diagnostic and medication information, compared to the ED visit. Although the patients diagnosed with epilepsy in the ED had received their diagnostic information prior to discharge from the ED, for most of them, it was not until the neurology follow up visit where they felt they had received the official diagnosis. A few participants some still had hesitancy toward their healthcare providers even after the outpatient follow up; they were nervous about trusting the physician and any recommendations that were made, and were frustrated and did not feel as though they were receiving adequate explanation of what had happened to them.

I just remember thinking, I don't feel good about this. A, I don't know you, B, you don't know my child. I mean we had already been in the hospital twice and he had this bit of information on a computer somewhere and who knows if he was looking at it. So we were on Depakote and he came off if for twenty-four hours because it was such a bad call. It was so horrible for him and I don't remember how we got through this first two weeks because it was terrifying. It was absolutely terrifying.

\section{Discussion}

First time seizure patients in the ED experience cognitive difficulties, fear, stigma and embarrassment, and uncertainty. Regardless of what diagnostic testing or medication prescriptions patients are given in the ED, patients trust medical professionals to act in their best interest during this time. Patients are generally satisfied with the care received in the ED, whether or not they had an EEG and early diagnosis and treatment, because they trust the healthcare providers to provide them with the current standard of care. Those who receive this testing in the ED, however, report alleviation of anxiety and reduced risk of future seizures or complications. All patients have expectations of rapid testing, diagnosis, and treatment. Performing medical tests, such as an EEG, in the ED can offer patients an explanation for their seizures and offer peace of mind for patients and families. An examination of patient experiences and patient-centered outcomes for first time seizure patients in the Emergency Department shows a divergence between clinical and therapeutic expectations and patient and family expectations. In fact, while clinical considerations for conducting an EEG in the ED for first time seizure patients are ambivalent, patient outcomes considerations for testing in the ED seem to be much clearer. Focus group members state that ED EEG performance may result in quicker initiation of anticonvulsant treatment, aids patients in expediting close follow up with an outpatient neurologist, decreases patient risk for further seizures, and reduces the number of patients who are lost to follow up. Additionally, those who received EEG testing report alleviation of anxiety and perceived reduced risk of future seizures or complications. 
However, there is some ambivalence expressed for ED EEG testing. It appears that clinical indications in favor of EEG testing -earlier and more definitive diagnosis, earlier treatment of potential epilepsy and resulting higher treatment efficacy, lessened chance of subsequent seizure episodes - are fairly evenly balanced with clinical reasons for not conducting EEG testing in the ED - lower treatment compliance because of reduced patient mental capacity and compromised memory, lack of opportunity for lengthy discussion between patient and physician, and the moderate rate of positive epilepsy diagnosis among first time seizure patients, and our focus group findings bear this out. However, when patient and family experience is taken into account, the argument for EEG testing is much stronger. Patient and family fear and uncertainty are alleviated through earlier testing and diagnosis and earlier presentation of health information. The desire on the part of the patients and their family members for rapid diagnosis, explanation, and treatment leads to a bias towards desiring EEG testing in the ED, especially because it is felt to expedite outpatient follow-up. Nonetheless, coupled with the earlier testing is a strong need for detailed (oral and written) health information to be provided to patients and families in the ED, and for rapid (within two weeks) follow up with a specialist who can review diagnosis and treatment options with the patient and family.

Also of particular consideration is that, regardless of what recommendation the ED staff makes - to conduct EEG testing in the ED or not - patients will be satisfied with the recommendation because of a positive ramification of physician hegemony - the belief that if the physician recommends a course of treatment, it must be the standard of care and can be trusted to be the best option for the situation.

Despite the patient-centered outcomes mandate of the ACA, shared decision making can only occur in a climate of two-way information and knowledge sharing. The cognitive impairments association with seizures makes that requirement difficult if not impossible to achieve in the ED at or close to the time of onset. Yet, ED providers' knowledge and understanding of the fear; confusion; and desire for rapid diagnosis, explanation, and treatment on the part of the patient leads to a bias towards recommending the earlier testing, diagnosis, and treatment associated with EEG testing in the ED. Coupled with the earlier testing, however, is a strong need for detailed (oral and written) health information to be provided to patients and families in the ED and for rapid (within two weeks) follow up with a specialist who can review diagnosis and treatment options with the patient and family.

\section{Conclusions}

In this paper, we suggest that obtaining and interpreting an EEG in the ED could expedite patient work-up, treatment, and disposition. In addition, as we suggest in this paper, earlier diagnosis and treatment could possibly ease anxiety and improve patients' experience.

In summary, adult seizure patients have difficulty remembering and understanding the events surrounding their ED visit due to their medical state at that time. Patients and their family members trust medical professionals to act in their best interest during this time, and trust that health care providers will perform the standard of care for evaluating first time seizures. While patients acknowledge reasons for not performing ED EEGs, overall they express partiality for performing EEGs in the ED, because they can result in quicker initiation of testing and anticonvulsant treatment, aid patients in expediting close follow up with a neurologist, may decrease risk for further seizures, and reduce the number of patients who are lost to follow up. Performing EEGs in the ED and initiating appropriate anticonvulsant therapy for those patients who are at high risk for future seizures expedites appropriate follow up care, decreases risk to patients and society, and offers patients a sense of security and control over their medical condition. In addition, performing medical tests such as an EEG in the ED can offer patients an explanation for their medical state and offer peace of mind for the patient and his/her family.

\section{References}

1. Biraben A, Taussig D, Thomas P, et al. Fear as the main feature of epileptic seizures. J Neurol Neurosurg Psychiatry 2001;70:186-91.

2. Ryan S, Räisänen U. The brain is such a delicate thing: an exploration of fear and seizures among young people with epilepsy. Chronic Illn 2012;8:214-24.

3. Weinstein S. Seizures and teens: the impact of seizures and epilepsy on families. Exceptional Parent 2007;37:61-5.

4. ACEP Clinical Policies Committee, Clinical Policies Subcommittee on Seizures. Clinical policy: critical issues in the evaluation and management of adult patients presenting to the emergency department with seizures. Ann Emerg Med 2004;43:605-25.

5. Krumholz A, Wiebe S, Gronseth GS, et al. Evidence-based guideline: management of an unprovoked first seizure in adults: report of the guideline development subcommittee of the American Academy of Neurology and the American Epilepsy Society. Neurology 2015;84:1705-13.

6. Huff JS, Melnick ER, Tomaszewski CA, et al. Clinical policy: critical issues in the evaluation and management of adult patients presenting to the emergency department with seizures. Ann Emerg Med 2014;63:437-47.

7. Hauser WA, Shinnar S, Berg AT et al. Seizure recurrence after a first unprovoked seizure: an extended follow-up. Neurology 1990;40:1163-70.

8. Eisner R, Turnbull T, Howes D, Gold I. Efficacy of a standard seizure workup in the ED. Ann Emerg Med 1986; 15:33-9.

9. King M, Newton M, Jackson G, et al. Epileptology of the first-time seizure presentation: a clinical, electroencephalographic, and magnetic resonance imaging study of 300 consecutive patients. Lancet 1998;352:1007-11. 
10. Wyman A, Mayes B, Hernandez-Nin J, et al. The First Time Seizure (FITS) ED EEG study. Acad Emerg Med 2015;22:S138.

11. Zehtabchi S, Baki SGA, Omurtag A, et al. Effect of microelectroencephalogram on clinical management and outcomes of emergency department patients with altered mental status: a randomized controlled trial. Acad Emerg Med 2014;21:283-91.

12. Praline J, Grujic J, Corcia P, et al. Emergent EEG in clinical practice. Clin Neurophysiol 2007;118:2149-55.

13. Sadleir L, Ingrid S. Optimizing electroencephalographic studies for epilepsy diagnosis in children with new-onset seizures. Archiv Neurol 2010;67:1345-9.

14. Kadambi P, Hart KW, Adeoye OM, et al. Electroencephalography findings in patients presenting to the ED for the evaluation of seizures. Am J Emerg Med 2015;33:100-3.

15. Ziai WC, Schlattman D, Llinas R, et al. Emergent EEG in the emergency department in patients with altered mental states. Clin Neurophysiol 2012;123:910-7.

16. Vranceanu A, Cooper C, Ring D. Integrating patient values into evidence-based practice: effective communication for shared decision-making. Hand Clin 2009;25:83-96.

17. Barratt A, Evidence based medicine and shared decision making: the challenge of getting both evidence and preferences into health care. Patient Educ Couns 2008;73:407-12.

18. Becker ER, Hockenberry JM, Bae J, et al. Factors in patients' experience of hospital care: evidence from California, 2009-2011. Patient Exp J, 2014:95-110.

19. Burns J. What works best for patients? PCORI hopes to pro- vide answers. Managed Care Mag Online 2012. Available from: http://www.managedcaremag.com.

20. Newhouse R, Barksdale DJ, Miller JA. The patient-centered outcomes research institute: research done differently. Nursing Res 2015;6:72-7.

21. Sequist TD, Taveras EM. Clinic-community linkages for high-value care. N Engl J Med 2014;371:2148-50.

22. Lee EO, Emanuel EJ. Shared decision making to improve care and reduce costs. N Engl J Med 2013;368:6-8.

23. Brodt A, Norton CK, Kratchman A. So much more than a 'pair of brown shoes': triumphs of patient and other stakeholder engagement in patient-centered outcomes research. Patient Exp J 2015;2:43-9.

24. Asbury J, Overview of focus group research. Qual Health Res 1995;5:414-20.

25. Carey MA. The group effect in focus groups: planning, implementing, and interpreting focus group research. In: Morse $\mathrm{JM}$, ed. Critical issues in qualitative research methods. Thousand Oaks, CA: Sage; 1994. pp 234-41.

26. Davis CS. Focus groups: applying communication theory through design, facilitation and analysis. New York, NY: Routledge; 2017.

27. Krueger RA. Moderating focus groups. Thousand Oaks, CA: Sage; 1998.

28. Charmaz K. Grounded theory: objectivist and constructivist methods. In: Denzin NK, Lincoln YS, eds. Handbook of qualitative research. Thousand Oaks, CA: Sage; 2000. pp 509-35.

29. Davis CS, Lachlan K. Straight talk about communication research methods. 3rd ed. Dubuque, IA: Kendall-Hunt. 2017. 